\title{
Everolimus y astrocitoma subependimario de células gigantes con degeneración quística
}

\author{
Everolimus and subependymal giant cell astrocytoma with cystic degeneration
}

\author{
- Carlos Emilio Restrepo', Andrés Felipe Cardona ${ }^{2,4}$, Luis Carlos Mayor ${ }^{5}$, Enrique Jiménez ${ }^{1,4}$ \\ 'Departamento de Neurocirugía, Fundación Santa Fe de Bogotá (Bogotá, Colombia). \\ ¿Grupo Oncología Clínica y Traslacional, Instituto de Oncología, Fundación Santa Fe de Bogotá (Bogotá, Colombia). \\ Fundación para la Investigación Clínica y Molecular Aplicada del Cáncer (FICMAC); investigador asociado ONCOLGroup. \\ ${ }^{4}$ Investigador asociado Red Latino Americana de Neuro-Oncología (RedLANO). \\ 'Departamento de Neurología, Sección Epileptología, Fundación Santa Fe de Bogotá (Bogotá, Colombia).
}

\begin{abstract}
Resumen
El complejo esclerosis tuberosa (CET) se caracteriza por la presencia de hamartomas en múltiples órganos, incluyendo el cerebro. Entre el $5 \%$ y $20 \%$ de quienes lo padecen presentan astrocitomas subependimarios de células gigantes (SEGA, por su sigla en inglés), hallazgo que se asocia a una elevada tasa de morbimortalidad atribuible a hidrocefalia obstructiva. El manejo clásico de los SEGA consiste en la resección quirúrgica, intervención que se relaciona en la actualidad a medicamentos que modulan la vía de señalización mTOR. Experimentos clínicos recientes han demostrado una reducción consistente del volumen tumoral, lo que favorece el control de las crisis epilépticas, hecho que mejora la calidad de vida. A continuación, se presenta el caso de un paciente de 16 años con diagnóstico de CET, a quien se documentó un SEGA con degeneración quística tratado después de dos resecciones quirúrgicas secuenciales con everolimus; dicho manejo permitió una respuesta parcial máxima asociada a la desaparición de otras manifestaciones neurológicas. La utilidad de medicamentos dirigidos a modular blancos moleculares específicos representa una opción terapéutica real en pacientes con el CET que presentan SEGA.
\end{abstract}

Palabras clave: Complejo esclerosis tuberosa, astrocitoma subependimario de células gigantes, mTOR, everolimus, hamartina, tuberina.

\begin{abstract}
The tuberous sclerosis complex (TSC) is characterized by the presence of hamartomas in many organs, including the brain; $5 \%$ to $20 \%$ of those suffering from TSC present subependymal giant cell astrocytoma (SEGA), such finding being associated with a high rate of morbimortality attributable to obstructive hydrocephalus. The classical management of SEGAs consists of surgical resection, such intervention being currently associated with drugs modulating the mTOR signalling pathway. Recent clinical trials have demonstrated that consistent tumor volume reduction has promoted the control of epileptic crises, thereby improving the quality of life. The case of a 16-year-old TSC patient is presented below who was diagnosed with SEGA accompanied by cystic degeneration treated with everolimus following two sequential surgical resections; such management led to a maximum partial response associated with the disappearance of other neurological manifestations. The efficacy of drugs directed to modulate specific molecular targets represent a real treatment option for patients with tuberous sclerosis and SEGA.
\end{abstract}

Key words: Tuberous sclerosis complex, subependymal giant cell astrocytoma, mtor, everolimus, hamartin, tuberin.

\section{Introducción}

El complejo esclerosis tuberosa (CET), también conocido como síndrome de Bourneville Pringle o Epiloia, es una enfermedad hereditaria autosómica dominante con penetrancia incompleta presente en 1 de cada 6.000 nacidos vivos. Con frecuencia produce lesiones en la retina (hamartomas astocíticos o facomas), piel (angiofibromas y máculas hipomelanóticas), pulmones (linfangioleiomiomatosis), riñones (angiomiolipomas) y en el corazón (rabdomiomas). De igual forma, suele afectar al sistema nervioso central, donde genera cambios descritos desde 18621-3; la manifestación neurológica más común es la epilepsia, que se presenta en el 70\% al $80 \%$ de los pacientes y que se relaciona de modo regular con la existencia de alteraciones cognitivas que incluyen el retraso en el desarrollo psicomotor, diversos trastornos del aprendizaje o neurocomportamentales tipo autista. Estos hallazgos se relacionan de manera directa o indirecta con la presentación de tuberomas 
corticales, heterotopias de la sustancia blanca y nódulos subependimarios, alteraciones que se caracterizan por una patogénesis común asociada a cambios en la migración y diferenciación anormal de las células embriónicas localizadas en la región subventricular2-4.

Es usual encontrar lesiones subependimarias de carácter nodular, que evolucionan en el tiempo hasta generar SEGA. Adriansen y colaboradores describieron, a partir de un estudio de corte transversal que incluyó 285 pacientes con el CET, la evidencia de estas neoplasias en el $20 \%$ de los casos, con un diámetro promedio al momento del diagnóstico de $11.4 \mathrm{~mm}( \pm 4.2 \mathrm{~mm})$ y la presencia de hidrocefalia en un $14 \% 5$.

La gran mayoría de los SEGA se acompañan de otros nódulos subependimarios que no exhiben de forma constitutiva la pérdida de heterocigocidad ni la autofosforilación proteica de la vía mTOR (mammalian target of rapamycin) ${ }^{6}$. Desde la perspectiva clínica, es importante distinguir los nódulos no neoplásicos de los SEGA, debido a la influencia sobre las intervenciones terapéuticas; por el momento, los criterios imaginológicos que se asocian con mayor agresividad son el tamaño tumoral, la localización de la lesión y su potencial para obstruir el drenaje normal del líquido cefalorraquídeo, la intensidad de señal en las secuencias T1 y la velocidad de crecimiento 7,8 .

El origen de las lesiones tumorales está vinculado a la activación de las mutaciones en los genes TSC1 y 2, cuyos productos derivados de la transcripción son la hamartina y tuberina, proteínas encargadas de promover la hidrólisis de GTP a través de una GTPasa del Rheb. Varios estudios han revelado que este complejo juega un papel esencial en el control del tamaño celular de la glía a través de la expresión del receptor para el factor de crecimiento similar a la insulina tipo 1 (IGFR1). Otros genes encargados de favorecer la progresión tumoral de los SEGA son el LKB1 (liver kinase B1) y el PTEN, los cuales conllevan mutaciones secundarias que favorecen la activación del factor derivado de la hipoxia tipo 1 (HIF1) y la supresión del p539-11.

Clásicamente, el manejo de los SEGA estuvo circunscrito a la cirugía y a la radioterapia. En el pasado, hasta el $70 \%$ de los pacientes eran operados de urgencia con signos de hipertensión endocraneana y morbilidad a largo plazo, sin embargo, Kruger y colaboradores introdujeron el uso de nuevas estrategias farmacológicas dirigidas contra la cascada derivada del mTOR (rapamicina y everolimus) $)^{12}$. A continuación, se presenta el caso de un paciente con SEGA tratado con everolimus después de dos exéresis parciales.

\section{Descripción del caso}

Se trata de un paciente de 16 años de edad con el CET diagnosticado a los 14 meses por la presencia de máculas hipomelanóticas, angiofibromas faciales y crisis mioclónicas manejadas con vigabatrina. En la primera resonancia magnética nuclear (RMN), tomada a los 17 meses de edad, se identificó la presencia de múltiples tuberomas corticales y nódulos subependimarios en los ventrículos laterales, especialmente, uno en el cuerno frontal izquierdo adyacente al foramen de Monro de $0.9 \times 1.0 \times 0.6 \mathrm{~cm}$ (figura $1 \mathrm{C}$ y tabla $1 \mathrm{~A}$ ), corroborando así el diagnóstico de la enfermedad. Por el adecuado control de la epilepsia, se suspendió el medicamento cuatro años después de su inicio; sin embargo, durante el año 2008, el paciente debutó con un nuevo episodio, para lo cual retomó la vigabatrina. Luego de dos meses, las crisis se hicieron más frecuentes, requiriendo el aumento de la dosis y la adición de lamotrigina y levetiracetam (tabla 1D-F).

En las imágenes de control realizadas en el 2010 (figura 1D-F), se identificó el crecimiento progresivo del nódulo subependimario del cuerno frontal izquierdo, que medía inicialmente $0.8 \mathrm{~cm}^{3}$ y alcanzó 5.6 $\mathrm{cm}^{3}$ (tabla 1B-E). Dicha modificación en el volumen condicionó una obstrucción parcial del foramen de Monro, razón por la cual se decidió llevar a cirugía en junio del 2010, logrando una resección parcial de la lesión; el reporte de la patología fue compatible con un SEGA. A pesar del manejo farmacológico, el paciente persistió con un pobre control de las crisis, por lo que el esquema se cambió incluyendo ácido valproico por el levetiracetam.

En la RMN de control hecha en diciembre del 2010, se halló crecimiento de la lesión, especialmente en su componente quístico (tabla 1G), que ya se visualizaba en el estudio imaginológico previo (tabla 1F). Sin embargo, por la estabilidad clínica, se decidió continuar el seguimiento. Para mayo del 2011, el paciente se tornó sintomático con indicios de hipertensión endocraneana, por lo cual se tomó una RMN que mostró un aumento de la lesión con evidencia radiológica de hidrocefalia y extensión al tercer ventrículo (figura 1G-I y tabla 1H), 

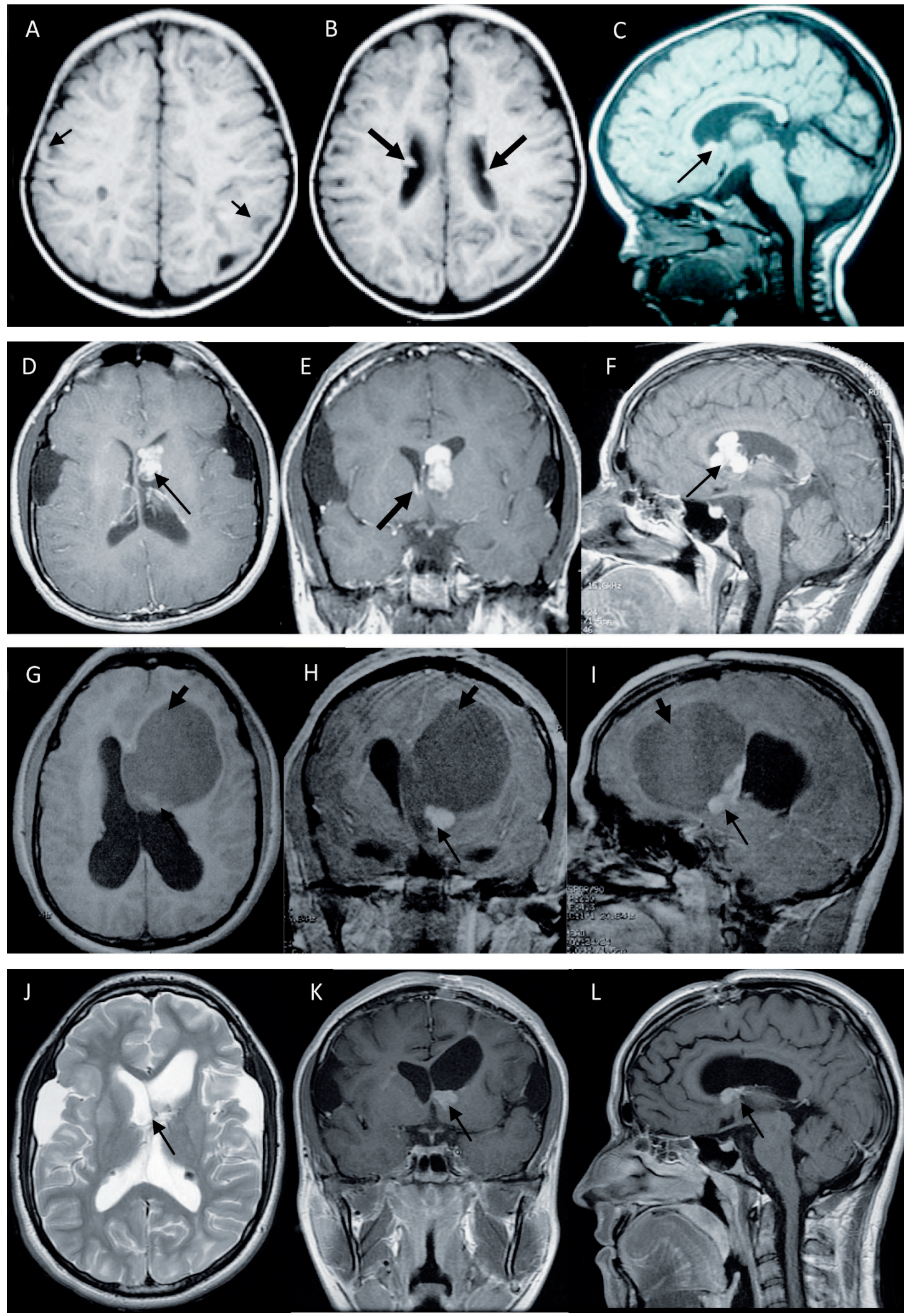

Figura 1. RMN cerebral durante el período de observación radiológica. Esta muestra la evolución del SEGA a partir de un nódulo subependimario a los 17 meses (A-C) y 16 años (D-F). En el período quirúrgico, se evidencia el crecimiento del componente sólido y la degeneración quística del SEGA tras el primer posoperatorio en el 2011 (G-I). En el período de manejo farmacológico con el inhibidor mTOR (everolimus), las imágenes control a los 5 meses $(\mathrm{J}-\mathrm{L})$ demuestran la disminución del componente sólido, no hay evidencia del componente quístico. Túberes $(\rightarrow)$, nódulos subependimarios $(\longrightarrow)$, SEGA sólido $(\rightarrow)$, SEGA quístico $(\rightarrow)$. 
Tabla 1. Evolución de la enfermedad y medicación relacionada con el control de las crisis epilépticas

\begin{tabular}{|c|c|c|c|}
\hline & Fecha RMN/(edad) & Volumen & Medicamento \\
\hline & Observación & Observación & Observación \\
\hline A & Abril 1995/(17 meses) & $0.9 \times 1 \times 0.6 \mathrm{~cm}=0.27 \mathrm{~cm}^{3}$ & Vigabatrina (14 meses a los 5 años) \\
\hline B & Octubre 2000/(6 años) & $1 \times 1.6 \times 1 \mathrm{~cm}=0.8 \mathrm{~cm}^{3}$ & No \\
\hline C & Julio 2004/(10 años) & $1.8 \times 2 \times 1 \mathrm{~cm}=1.8 \mathrm{~cm}^{3}$ & No \\
\hline D & Septiembre 2008/(14 años) & $2.1 \times 2 \times 1 \mathrm{~cm}=2.1 \mathrm{~cm}^{3}$ & $\begin{array}{l}\text { Vigabatrina } 1.000 \text { mg/día } \\
\text { Vigabatrina } 2.000 \text { mg/día } \\
\text { Lamotrigina } 100 \text { mg/día }\end{array}$ \\
\hline $\mathbf{E}$ & Abril 2010/(16 años) & $2.5 \times 2.5 \times 1.8 \mathrm{~cm}=5.6 \mathrm{~cm}^{3}$ & $\begin{array}{l}\text { Vigabatrina } 4.000 \mathrm{mg} / \mathrm{día} \\
\text { Lamotrigina } 500 \mathrm{mg} / \mathrm{dí}^{\prime} \\
\text { Levetiracetam } 1.500 \mathrm{mg} / \mathrm{día}\end{array}$ \\
\hline & Cirugía & Cirugía & Cirugía \\
\hline $\mathbf{F}$ & $\begin{array}{c}\text { Julio 2010/(16 años) } \\
\text { (Primer posoperatorio) }\end{array}$ & Sólido: $\begin{aligned} 2.6 \times 1.5 \times 1.7 \mathrm{~cm} & =3.3 \mathrm{~cm}^{3} \text { quiste: } 3 \times 2.1 \times 3.6 \mathrm{~cm} \\
& =11.3 \mathrm{~cm}^{3}\end{aligned}$ & $\begin{array}{l}\text { Vigabatrina } 4.000 \mathrm{mg} / \text { día } \\
\text { Lamotrigina } 500 \mathrm{mg} / \mathrm{día} \\
\text { Levetiracetam } 1.500 \mathrm{mg} / \mathrm{día}\end{array}$ \\
\hline G & Diciembre 2010/(17 años) & $\begin{array}{l}\text { Sólido: } 3 \times 1.8 \times 1.6 \mathrm{~cm}=4.32 \mathrm{~cm}^{3} \\
\text { Quiste: } 5 \times 6.4 \times 3.9 \mathrm{~cm}=6.2 \mathrm{~cm}^{3}\end{array}$ & $\begin{array}{l}\text { Vigabatrina } 3.500 \text { mg/día } \\
\text { Lamotrigina } 300 \text { mg/día } \\
\text { Ácido valproico } 500 \text { mg/día }\end{array}$ \\
\hline H & 3 de mayo 2011/(17 años) & $\begin{array}{l}\text { Sólido: } 3.4 \times 2 \times 1.8 \mathrm{~cm}=6.12 \mathrm{~cm}^{3} \\
\text { Quiste: } 6 \times 7 \times 5 \mathrm{~cm}=10.5 \mathrm{~cm}^{3}\end{array}$ & $\begin{array}{l}\text { Vigabatrina } 1.500 \mathrm{mg} / \text { día } \\
\text { Lamotrigina } 300 \mathrm{mg} / \text { día } \\
\text { Ácido valproico } 500 \mathrm{mg} / \text { día }\end{array}$ \\
\hline I & $\begin{array}{l}7 \text { de mayo } 2011 /(17 \text { años) } \\
\text { (Segundo posoperatorio) }\end{array}$ & $\begin{array}{c}1.6 \times 2.2 \times 2.4 \mathrm{~cm}=4.4 \mathrm{~cm} 3 \text { Técnica de volumetría 3D: } \\
4.9 \mathrm{~cm}^{3}\end{array}$ & $\begin{array}{c}\text { Vigabatrina } 1.500 \text { mg/día } \\
\text { Lamotrigina } 300 \text { mg/día } \\
\text { Ácido valproico } 500 \text { mg/día } \\
\text { Losartán } 25 \text { mg/día }\end{array}$ \\
\hline & Inhibidor mTOR & Inhibidor mTOR & Inhibidor mTOR \\
\hline J & $\begin{array}{l}\text { Septiembre } 2011 /(17 \text { años) } \\
\text { (Control } 3 \text { meses) }\end{array}$ & $1.1 \times 1.5 \times 1.3 \mathrm{~cm}=1.07 \mathrm{~cm}^{3}$ & $\begin{array}{l}\text { Vigabatrina } 500 \text { mg/día } \\
\text { Lamotrigina } 300 \text { mg/día } \\
\text { Ácido valproico } 250 \text { mg/día } \\
\text { Everolimus } 5 \text { mg/día } \\
\text { Losartán } 25 \text { mg/día }\end{array}$ \\
\hline $\mathbf{K}$ & $\begin{array}{l}\text { Diciembre 2011/(18 años) } \\
\text { (Control } 5 \text { meses) }\end{array}$ & $\begin{array}{c}1 \times 1.4 \times 1.38 \mathrm{~cm}=0.96 \mathrm{~cm}^{3} \text { Técnica de volumetría 3D: } \\
0.97 \mathrm{~cm}^{3}\end{array}$ & $\begin{array}{c}\text { Vigabatrina } 500 \text { mg/día } \\
\text { Lamotrigina } 300 \mathrm{mg} / \text { día } \\
\text { Acido valproico } 250 \mathrm{mg} / \text { día } \\
\text { Everolimus } 5 \mathrm{mg} / \mathrm{dí} \\
\text { Amlodipino } 5 \mathrm{mg} / \mathrm{día} \\
\text { Losartán } 25 \mathrm{mg} / \mathrm{dí} \\
\text { Atorvastatina } 20 \mathrm{mg} / \mathrm{día}\end{array}$ \\
\hline
\end{tabular}

Correlación entre el volumen del SEGA, el tiempo y los requerimientos de medicación. Período de observación radiológica (A-E), período quirúrgico (F-l) y período de manejo farmacológico con inhibidor mTOR (everolimus) (J-K).

razón por la que se llevó por segunda ocasión a cirugía, consiguiendo la resección completa del quiste, pero dejando un remanente sólido (tabla 11).

Ante la recurrencia de la lesión, se decidió iniciar everolimus $5 \mathrm{mg} / \mathrm{día}$ ( $3 \mathrm{mg} / \mathrm{m}^{2} / \mathrm{día}$ ) a partir de junio del 2011. En el primer control, llevado a cabo tras completar tres meses de la medicación, se encontró resolución completa de las crisis epilépticas, lo que permitió la disminución progresiva del tratamiento. Igualmente, se identificó una regresión de los angiofibromas faciales y se documentó en la RMN de control una reducción significativa del tamaño del SEGA sin evidencia alguna del componente quístico (el 79\% por técnica de volumetría 3D y el 76\% por medición de la elipsoide) (tabla 1J).

El paciente ha mostrado buena tolerancia al medicamento, excepto por un episodio de estomatitis autolimitada, hipercolesterolemia e hipertensión arterial, eventos adversos manejados con losartán y atorvastatina. Para el siguiente control (cinco meses después de iniciar el inhibidor mTOR), se mantuvo la mejoría clínica y la regresión de la lesión, que ahora presenta un volumen de $0.97 \mathrm{~cm}^{3}$ (figura 1J-L y tabla 1K). De igual forma, el entorno familiar refiere una recuperación subjetiva del rendimiento académico.

\section{Discusión}

La principal causa de muerte en pacientes con el CET está relacionada con diversas complicaciones neurológicas. Los SEGA pueden generar muerte súbita secundaria a hidrocefalia obstructiva, por lo cual se recomienda llevar a cabo imágenes y un seguimiento periódico (cada seis meses a un año), con el objetivo de documentar cualquier variación en el crecimiento 8,9,13,14. Aunque se considera al SEGA como una lesión de crecimiento lento, existen algunos criterios que ayudan a decidir cuándo efectuar una cirugía antes de que el paciente se torne sintomático. Ribaupierre y colaboradores propusieron que las lesiones mayores de $5 \mathrm{~mm}$ próximas al foramen de Monro y con calcificación incompleta en las que se demuestre un aumento progresivo del tamaño en dos imágenes consecutivas deben ser llevadas a cirugía ${ }^{8}$. 
La resección quirúrgica macroscópica asegura un adecuado control de la lesión en la mayoría de los casos, no obstante, solo se logra en el $20 \%$, debido a la relación con estructuras interventriculares, como el núcleo caudado, el epéndimo y el fórnix ${ }^{8,15}$. Cuando no se logra una exéresis completa, existe un alto riesgo de recurrencia y la consecuente necesidad de otras intervenciones quirúrgicas; sin embargo, también existe la posibilidad de que la lesión tumoral permanezca estable, especialmente en la adultez ${ }^{16}$. Nuestro paciente representa el ejemplo clásico de la progresión de la enfermedad y lo que implicó su manejo quirúrgico; durante la niñez, hubo un crecimiento lento de uno de los nódulos subependimarios adyacentes al foramen de Monro, y, al entrar a la adolescencia, se documentó un aumento rápido de la lesión con obstrucción parcial asociada a exacerbación de las crisis epilépticas, razón por la cual se recurrió a la cirugía sin lograr una resección completa, evento que predispuso a la progresión.

Una situación particular reportada en este caso es la presencia de un componente quístico, el cual es infrecuente ${ }^{17}$; este progresa en conjunto con la porción sólida, tornándose sintomático. Aunque el manejo quirúrgico es la primera opción en este tipo de lesiones, conlleva riesgos como la hemiparesia (transitoria 12\% y permanente $6 \%)^{18}$, disminución de la agudeza visual $(20 \%)^{16}$ y alteraciones cognitivas y de la memoria $(5 \%)^{8}$, o generales, como el sangrado $(13 \%)^{18}$, la infección $(20 \%)^{19} \mathrm{e}$, incluso, la muerte $(13 \%)^{18}$.

EI CET es causado por mutaciones que inactivan los genes TSC1 y 2; el primero de ellos, localizado en el cromosoma 9p34, mide $45 \mathrm{~Kb}$ y está dividido en 23 exones que codifican un mARN de $8.6 \mathrm{~Kb}$ con un producto funcional denominado hamartina, una proteína de 1.164 aminoácidos. Se han descrito diversos tipos de mutaciones desde los cambios puntuales en una base hasta extensas deleciones. El gen TSC2 está ubicado en el segmento $16 \mathrm{p} 13$, mide $43 \mathrm{~Kb}$ y está dividido en 41 exones que codifican la tuberina, una proteína de 1.807 aminoácidos que presenta heterogeneidad mutaciona ${ }^{20,21}$. La hamartina y la tuberina interactúan al formar un complejo funcional que interrumpe el proceso de señalización de la vía mTOR, inhibe la PI3K (fosfatidil inositol-3-kinasa) y la activación de señales dependientes de factores de crecimiento derivados de la insulina (IGF1 e IGF2).

Así mismo, interactúa con otros genes, como AKT1,
CLIP1, GPHN, P70-S6 kinasa, RHEB, RICTOR, STAT1 y STAT3 ${ }^{21,22,23}$. Además, activa la GTPasa de RHEB, que, a su vez, regula a mTOR, la cual está involucrada en el crecimiento, proliferación, síntesis proteica, metabolismo y supervivencia celular 2,3,9. Por todas estas consideraciones, parecía claro que mTOR constituye un blanco farmacológico para el control del CET. En la actualidad, existen dos inhibidores de este complejo aprobados internacionalmente para el uso en humanos, la rapamicina (sirolimus) y el RAD001 (everolimus). La utilidad de estos compuestos se ha probado en estudios preclínicos y experimentos fase I y II, dando como resultado el control y la disminución en el tamaño de los hamartomas encontrados en diferentes órganos de los pacientes con el CET ${ }^{14}$. En el Trial of rapamycin therapy for patients with tuberous sclerosis complex and sporadic $L A M$, se demostró la disminución de los angiomiolipomas en el 53\%, aparte de una mejoría significativa en las pruebas de función pulmonar ${ }^{24}$.

De manera similar, el Tesstal documentó una disminución en los angiomiolipomas renales del 50\% después de 12 meses de tratamiento ${ }^{25}$. Otros han descrito la retracción de los rabdomiomas cardíacos después de la administración de everolimus ${ }^{26}$ y la notable mejoría de las lesiones cutáneas utilizando preparaciones tópicas de los inhibidores mTOR ${ }^{27,28}$. Cabe resaltar que en nuestro caso el paciente ha tenido una disminución progresiva de los angiomiolipomas faciales luego del inicio del everolimus. Del mismo modo en que se ha estudiado el uso de los inhibidores mTOR para el manejo de los angiomiolipomas, varios estudios han reportado su utilidad en los pacientes con SEGA. Franz y colaboradores informaron, en el 2006, el uso del sirolimus en cinco pacientes con el CET que presentaban SEGA; esta intervención permitió una reducción en el tamaño de las lesiones que osciló entre el $45 \%$ y $62 \%$ tras una mediana de tiempo para el tratamiento de 7.3 meses $^{29}$. En vista de estos resultados, entre el 2008 y 2010, se informaron cinco pacientes más, todos con una disminución del tamaño que varió entre el 37\% y 82\% después de 2 a 5 meses de exposición al sirolimus $30,31,32$.

Luego de la aprobación del everolimus como inmunomodulador en pacientes postrasplante renal y cardíaco, y para el tratamiento de sujetos con carcinoma renal de células claras, Yalon reportó el primer caso de un SEGA tratado exitosamente con este medicamento. Este correspondió a un paciente de 28 años con múltiples 
lesiones, sometido a varias cirugías, que, después de 11 meses de manejo con el inhibidor mTOR, obtuvo respuesta y mejoría clínica que se limitó debido a la presentación de hipertensión y aumento de la $\mathrm{CPK}^{33}$. Posteriormente, Krueger y colaboradores informaron los resultados del estudio de registro que incluyó 28 pacientes con una edad promedio de 11 años tratados con everolimus a una dosis inicial de $3 \mathrm{mg} / \mathrm{m}^{2}$ día ajustada según niveles cuantificados durante los primeros seis meses de tratamiento $(5-15 \mathrm{ng} / \mathrm{mm})$.

La mediana de duración de la intervención fue 21.5 meses y la disminución de los SEGA fue de al menos el $30 \%$ en 21 sujetos (75\%) y del 50\% en nueve pacientes (32\%); la reducción más notable se encontró en los primeros tres meses de intervención, especialmente en las lesiones de mayor tamaño, y los efectos adversos más comunes fueron las infecciones respiratorias del tracto superior, la estomatitis y la dislipidemia'2. Según nuestro conocimiento, el presente caso es el número 30 reportado en la literatura y describe la mejoría global de un paciente con el CET y SEGA quístico recurrente manejado con everolimus. En él, se registró una evidencia imaginológica de regresión tumoral del 79\% en los primeros tres meses con la presentación de una morbilidad conocida y manejable.

Un aspecto que se evaluó por primera vez de forma sistemática en el estudio de Krueger fue la mejoría de las crisis epilépticas, de la calidad de vida y del nivel cognitivo de los pacientes. En la actualidad, existe la hipótesis de que la vía mTOR está involucrada en la fisiopatología de la epileptogénesis y de que su hiperactivación conlleva la formación anormal de estructuras y sinapsis neuronales, así como la expresión alterada de neurotransmisores y de canales iónicos implicados en la epilepsia ${ }^{9}$. Hasta el momento, hay varios modelos que refuerzan esta teoría, entre otros, la presencia de crisis en ratones con inactivación en el gen TSC1, que es reversible con la administración de sirolimus y que regresa con la supresión del medicamento ${ }^{34,35}$. Frecuentemente, se cree que el déficit cognitivo y las alteraciones neuropsiquiatricas que se observan en el CET son atribuibles a la presencia de los túberes corticales y a la epilepsia, sin embargo, se presume que el $50 \%$ de los casos tienen esta asociación ${ }^{4}$.

Estudios en modelos murinos con pérdida de la funcionalidad por heterocigocidad de los genes TSC1 y 2 han encontrado cambios en la capacidad cognitiva en ausencia de epilepsia, túberes corticales o anomalías estructurales en las neuronas. Estos ratones tuvieron problemas de aprendizaje, memoria y ubicación visoespacial, al igual que alteraciones en el comportamiento social, hallazgos que mejoraron después de la administración de rapamicina ${ }^{36}$. Desde la perspectiva clínica, también documentaron una reducción significativa en la frecuencia de las crisis epilépticas, ya que, de los 16 pacientes en los que se reportó un electroencefalograma (EEG) de base, se halló que en 9 disminuyó la frecuencia del daño, en 6 no cambió y solo en 1 empeoró. Respecto de la calidad de vida, hubo una mejoría global sin lograr identificar cambios considerables en la perspectiva intelectual y en las evaluaciones neuropsicológicas ${ }^{12}$.

Recientemente, el mismo grupo informó un cambio relevante en la difusión de la materia blanca, sugiriendo que el defecto genético a nivel cerebral puede ser modificado farmacológicamente ${ }^{37}$. En el momento, se está desarrollando un estudio para determinar el impacto real del everolimus sobre las crisis epilépticas y el estado neurocognitivo de los pacientes con el CET (NCT01070316). En nuestro caso, hubo una mejoría completa de la epilepsia, lo que permitió el retiro progresivo de la medicación para este fin, así como la recuperación progresiva del rendimiento escolar sin que se hayan practicado pruebas neuropsicológicas pre y pos everolimus.

Un aspecto importante y hasta el momento no establecido en el tratamiento con inhibidores mTOR en CET es el tiempo de duración requerido para mantener los efectos terapéuticos ${ }^{39,40}$; de los primeros 5 casos reportados por Franz y colaboradores, 1 paciente presentó crecimiento de la lesión después de haber recibido rapamicina por cinco meses, requiriendo el reinicio del medicamento, con el que se obtuvo una segunda respuesta favorable a los 20 meses $^{29}$. Por otro lado, Krueger y colaboradores observaron una mediana de duración para el tratamiento de 21.5 meses (4.7-34.4), 27 de los 28 pacientes completaron el ciclo básico de seis meses, 2 de estos descontinuaron a los 12.5 y 21.5 meses, y otro paciente de los que había completado los criterios de reducción mayor del 75\% descontinuó el medicamento, documentándose un crecimiento de la lesión, por lo cual se reinición ${ }^{2}$. Nuestro paciente ha continuado el medicamento, logrando hasta el momento de la publicación un efecto terapéutico positivo sin 
mayor toxicidad, sin embargo, hace falta un seguimiento más prolongado para establecer la duración ideal y la estabilidad a largo plazo.

\section{Conclusiones}

La evidencia creciente acerca de la utilidad de medicamentos dirigidos a modular blancos moleculares específicos representan una opción terapéutica real en pacientes con el CET que presentan SEGA, no solo por el efecto que ejercen sobre el crecimiento del tumor, sino también como parte del control genético de la enfermedad, lo que permite controlar otras manifestaciones neurológicas, como la epilepsia y los hamartomas localizados en otros órganos. Los autores concuerdan con el algoritmo que propone que las lesiones subependimarias con criterios para SEGA o aquellas sintomáticas se traten con cirugía, siguiendo los casos con resección completa para identificar recurrencias tardías. Por otra parte, en quienes se logre una exéresis parcial se puede considerar una segunda intervención o el manejo farmacológico con everolimus ${ }^{15}$. Se requieren más estudios para valorar la efectividad de estos medicamentos utilizados de forma temprana o para el control de las crisis, incluyendo una evaluación integral de la toxicidad con el uso a largo plazo.

\section{Referencias}

1. Osborne JP, Fryer A, Webb D. Epidemiology of tuberous sclerosis. Ann N Y Acad Sci. 1991;615:125-7.

2. Crino $P B$, Nathanson $\mathrm{KL}$, Henske EP. The tuberous sclerosis complex. N Engl J Med. 2006;355(13):1345-56.

3. Curatolo $P$, Bombardieri $R$, Jozwiak $S$. Tuberous sclerosis. Lancet. 2008;372(9639):657-68

4. Ehninger D, de Vries PJ, Silva AJ. From mTOR to cognition: molecular and cellular mechanisms of cognitive impairments in tuberous sclerosis. J Intellect Disabil Res. 2009;53(10):838-51.

5. Adriaensen ME, Schaefer-Prokop $C M$, Stijnen $T$, Duyndam DA, Zonnenberg BA, Prokop M. Prevalence of subependymal giant cell tumors in patients with tuberous sclerosis and a review of the literature. Eur J Neurol. 2009;16(6):691-6.

6. Chan JA, Zhang H, Roberts PS, Jozwiak S, Wieslawa G, LewinKowalik J, et al. Pathogenesis of tuberous sclerosis subependymal giant cell astrocytomas: biallelic inactivation of TSC1 or TSC2 leads to mTOR activation. J Neuropathol Exp Neurol. 2004;63(12):1236-42.

7. Goh S, Butler W, Thiele EA. Subependymal giant cell tumors in tuberous sclerosis complex. Neurology. 2004;63(8):1457-61.

8. de Ribaupierre S, Dorfmüller G, Bulteau C, Fohlen M, Pinard $J M$, Chiron C, et al. Subependymal giant-cell astrocytomas in pediatric tuberous sclerosis disease: when should we operate? Neurosurgery. 2007;60(1):83-9.

9. Wong M. Mammalian target of rapamycin (mTOR) inhibition as a potential antiepileptogenic therapy: from tuberous sclerosis to common acquired epilepsies. Epilepsia. 2010;51(1):27-36.

10. Inoki K, Guan KL. Tuberous sclerosis complex, implication from a rare genetic disease to common cancer treatment. Hum Mol Genet. 2009;18(R1):R94-100.

11. Mieulet $\mathrm{V}$, Lamb RF. Tuberous sclerosis complex: linking cancer to metabolism. Trends Mol Med. 2010;16(7):329-35.

12. Krueger DA, Care MM, Holland K, Agricola K, Tudor C, Mangeshkar $\mathrm{P}$, et al. Everolimus for subependymal giant-cell astrocytomas in tuberous sclerosis. N Engl J Med. 2010;363(19):1801-11.

13. Ekici MA, Kumandas S, Per $H$, Ekici A, Tucer B, Gumus $H$, et al. Surgical timing of the subependymal giant cell astrocytoma
(SEGA) with the patients of tuberous sclerosis complex. Turk Neurosurg. 2011;21(3):315-24.

14. Sampson JR. Therapeutic targeting of $m T O R$ in tuberous sclerosis. Biochem Soc Trans. 2009;37(Pt 1):259-64

15. Berhouma M. Management of subependymal giant celltumors in tuberous sclerosis complex: the neurosurgeon's perspective. World J Pediatr. 2010;6(2):103-10

16. Kim SK, Wang KC, Cho BK, Jung HW, Lee YJ, Chung YS, et al. Biological behavior and tumorigenesis of subependymal giant cell astrocytomas. J Neurooncol. 2001;52(3):217-25.

17. Sugita $Y$, Taguchi A, Miyagi J, Yuge $T$, Tomita T, Shigemori $M$, et al. The cystic growth of a subependymal giantcell astrocytoma with tuberous sclerosis. Kurume Med J. 1992;39(2):123-8.

18. Cuccia V, Zuccaro G, Sosa F, Monges J, Lubienieky F, Taratuto AL. Subependymal giant cell astrocytoma in children with tuberous sclerosis. Childs Nerv Syst. 2003;19(4):232-43.

19. Kumar $R$, Singh $V$. Subependymal giant cell astrocytoma: a report of five cases. Neurosurg Rev. 2004;27(4):274-80.

20. Napolioni V, Moavero $R$, Curatolo P. Recent advances in neurobiology of Tuberous Sclerosis Complex. Brain Dev. 2009;31(2):104-13.

21. Young J, Povey S. The genetic basis of tuberous sclerosis. Mol Med Today. 1998;4(7):313-9.

22. Brugarolas J, Lei K, Hurley RL, Manning BD, Reiling JH, Hafen $E$, et al. Regulation of mTOR function in response to hypoxia by REDD1 and the TSC1/TSC2 tumor suppressor complex. Genes Dev. 2004;18(23):2893-904

23. Ellisen LW. Growth control under stress: mTOR regulation through the REDD1-TSC pathway. Cell Cycle. 2005;4(11):150002 .

24. Bissler JJ, McCormack FX, Young LR, Elwing JM, Chuck G, Leonard JM, et al. Sirolimus for angiomyolipoma in tuberous sclerosis complex or lymphangioleiomyomatosis. N Engl J Med. 2008;358(2):140-51.

25. Davies DM, Johnson SR, Tattersfield AE, Kingswood JC, Cox $J A$, McCartney DL, et al. Sirolimus therapy in tuberous scle- 
rosis or sporadic lymphangioleiomyomatosis. N Engl J Med. 2008;358(2):200-3.

26. Tiberio D, Franz DN, Phillips JR. Regression of a cardiac rhabdomyoma in a patient receiving everolimus. Pediatrics. 2011;127(5):e1335-7.

27. Hofbauer GF, Marcollo-Pini $A$, Corsenca A, Kistler AD, French $L E$, Wüthrich RP, et al. The mTOR inhibitor rapamycin significantly improves facial angiofibroma lesions in a patient with tuberous sclerosis. Br J Dermatol. 2008;159(2):473-5.

28. Mutizwa MM, Berk DR, Anadkat MJ. Treatment of facial angiofibromas with topical application of oral rapamycin solution $(1 \mathrm{mgmL}(-1))$ in two patients with tuberous sclerosis. Br J Dermatol. 2011;165(4):922-3

29. Franz DN, Leonard J, Tudor C, Chuck G, Care M, Sethuraman $G$, et al. Rapamycin causes regression of astrocytomas in tuberous sclerosis complex. Ann Neurol. 2006:59(3):490-8.

30. Koenig MK, Butler IJ, Northrup H. Regression of subependymal giant cell astrocytoma with rapamycin in tuberous sclerosis complex. J Child Neurol. 2008;23(10):1238-9.

31. Lam C, Bouffet $E$, Tabori $U$, Mabbott $D$, Taylor M, Bartels U. Rapamycin (sirolimus) in tuberous sclerosis associated pediatric central nervous system tumors. Pediatr Blood Cancer. 2010;54(3):476-9.

32. Birca A, Mercier C, Major P. Rapamycin as an alternative to surgical treatment of subependymal giant cell astrocytomas in a patient with tuberous sclerosis complex. J Neurosurg Pediatr. 2010;6(4):381-4.
33. Yalon M, Ben-Sira L, Constantini S, Toren A. Regression of subependymal giant cell astrocytomas with RAD001 (Everolimus) in tuberous sclerosis complex. Childs Nerv Syst. 2011;27(1):179-81.

34. Erbayat-Altay E, Zeng LH, Xu L, Gutmann D, Wong M. The natural history and treatment of epilepsy in a murine model of tuberous sclerosis. Epilepsia. 2007;48(8):1470-6.

35. Zeng LH, Xu L, Gutmann DH, Wong M. Rapamycin prevents epilepsy in a mouse model of tuberous sclerosis complex. Ann Neurol. 2008;63(4):444-53.

36. Ehninger D, Han S, Shilyansky C, Zhou Y, Li W, Kwiatkowski DJ, et al. Reversal of learning deficits in a Tsc2+/- mouse model of tuberous sclerosis. Nat Med. 2008;14(8):843-8

37. Tillema JM, Leach JL, Krueger DA, Franz DN. Everolimus alters white matter diffusion in tuberous sclerosis complex. Neurology. 2012;78(8):526-31

38. Pascual-Castroviejo I, Pascual-Pascual SI, Velázquez-Fragua R, Viaño J, Carceller F, Hernández-Moneo JL, et al. [Subependymal giant cell astrocytoma in tuberous sclerosis complex. A presentation of eight paediatric patients]. Neurología. 2010;25(5):314-21

39. Campen CJ, Porter BE. Subependymal Giant Cell Astrocytoma (SEGA) Treatment Update. Curr Treat Options Neurol. 2011;13(4):380-5.

40. Nawashiro H, Shinomiya N. Everolimus and giantcell astrocytomas in tuberous sclerosis. N Engl J Med. 2011;364(6):576-7. 